\title{
Study on the Online Control System to Prevent Drunk Driving Based on Photoelectric Detection Technology
}

\author{
Lu Liming ${ }^{1}$, Yang Yuchuan ${ }^{2}$, and Lu Jinfu ${ }^{1}$ \\ ${ }^{1}$ East China jiaotong university, Nanchang, 330013, P.R. China \\ luliming@ecjtu.jx.cn \\ ${ }^{2}$ Yichun Vocational Technical College, Yichun, 336000, P.R. China
}

\begin{abstract}
A drink driving online control system based on photoelectric detection technology is introduced in the paper. The system can take the initiative to force motor vehicle drivers to test their drinking status, and reasonably control the driver's behavior accordingly. The basic principle is to use the characteristic that $1185 \mathrm{~nm}$ wavelength IR is absorbed by alcohol the most easily to detect the motor vehicle driver's alcohol concentration and to determine the extent of motor vehicle drivers drinking. The alcohol concentration detected is converted into photoelectric signal that is handled by $89 \mathrm{C} 2051 \mathrm{MCU}$. The signal handled is exported to voice devices to get voice prompts and warning, and exported to the relay of ignition circuit system to cut off the ignition system to control the engine's starting and prevent the driver drunk driving, and exported to electro-hydraulic proportional flow valve to control the maximum speed of motor vehicle according to the driver's alcohol content. The purpose of this study is to be able to effectively solve the problem of drink driving and avoid serious traffic accidents resulting from drunken driving.
\end{abstract}

Keywords: Photoelectric Technology, Drunk Driving, Control System.

\section{Introduction}

The danger of drink driving motor vehicle is known as we all. "Drivers shall not drive a motor vehicle after drinking" is clearly stated in Article 2 of "The People's Republic of China Road Traffic Safety Law.", but some motor vehicle drivers have luck idea in their brain, resulting in the provisions existing in name only, causing a number of serious accidents. To avoid this incident happening, a drink driving online control system based on photoelectric detection technology is introduced in the paper.

\section{The Overall Program of Realizing the Online Detection Control System to Prevent Drunk Driving}

By a gas absorption device constituted by an airflow sensor and a temperature sensor, motor vehicle drivers are forced to detect their breath alcohol concentration before driving, to prevent them to avoid alcohol testing by ventilating through the windows and covering the test probes and other means. The test of alcohol concentration of 
motor vehicle drivers is done by the equipment detecting breath alcohol concentration based on IR spectroscopy. The optical signal obtained by the test is turned into electric signal by photoelectric detector and magnified by amplifier. The output signal of the alcohol concentration testing device and the output signal of the gas absorption device are respectively inputted control circuit device and processed by the 89C2051 MCU. The new signal got is exported to voice devices to get voice prompts and warning messages, exported to the ignition circuit of the start system to control the motor vehicle start, exported to electro-hydraulic proportional flow valve to limit vehicle maximum speed. The specific procedure is shown in figure 1 .

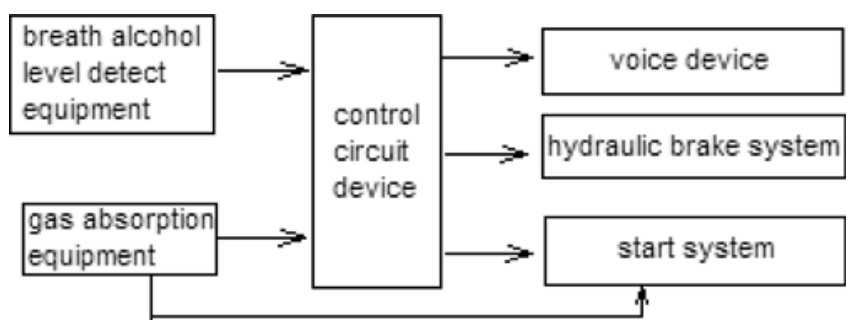

Fig. 1. The online detection and control system to prevent drunk driving

\section{The Gas Absorption Device to Force Motor Vehicle Drivers to Detect Their Breath Alcohol Concentration Before Driving}

The gas absorption device accepting the motor vehicle driver's breath gas mainly consists of a temperature sensor and an airflow sensor, to control the relay turning on and off the ignition system. The purpose of using the temperature sensor is to Identify whether the air is human body's gas. The basic principle of the temperature sensor generating electrical signal is to compare gas flow temperature and ambient temperature, because the gas temperature of human body breathing is higher than ambient temperature, to determine whether the electrical signal is generated. The purpose of using the airflow sensors also is to Identify whether the air is human body's gas. The basic principle of the airflow sensor generating electrical signal is to compare the gas flow rate of human body breathing and the flow rate of the environment air to determine whether the electrical signal is generated, because the gas flow of human body breath is faster than the gas flow of environment air in cab. When the two sensors generate signals at the same time, the motor vehicle driver's breath can be regarded as being valid to prevent the driver to avoid detection.

\section{The Alcohol Concentration Detector Based on Spectroscopy Technology}

It was found by experiment that the most IR wavelength absorbed by alcohol is $1185 \mathrm{~nm}$ [1], and the most IR wavelength absorbed by carbon dioxide is $1575 \mathrm{~nm}$, and the most IR wavelength absorbed by water vapor is $1315 \mathrm{~nm}$. Under normal 
circumstances the gas of human body exhale mainly contains of carbon dioxide and water vapor. The extent of motor vehicle drivers drinking can be determined by observing the absorbed situation of wavelength $1185 \mathrm{~nm}$ [1] IR when it passes through the gas region of the driver exhale. Testing device is shown in Figure 2. Using hollow cathode lamp to generate wavelength $1185 \mathrm{~nm}$ IR, when it passes through the gas region of drivers exhale, alcohol will absorb some of its spectral energy. Using wavelength selector further to filter out other wavelengths of the spectral, the remaining spectral energy is converted into electrical signals by the optical converter. The electrical signal is amplified by the amplifier and then is sent to the control circuit device.

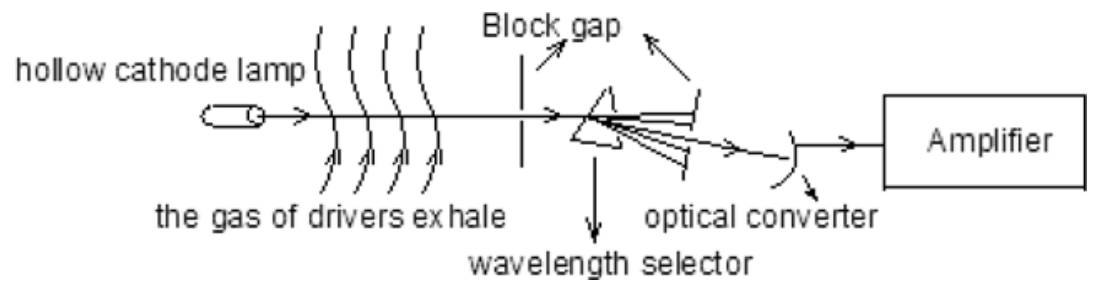

Fig. 2. Alcohol concentration detector

\section{Drunk Driving Control Circuit System}

The voltage of control circuit is $+5 \mathrm{~V}$. The control system mainly consists of 89C2051 MCU[2], MTP337A temperature sensors, speed sensor, gas flow sensor, voice prompt module, voice warning module, on-off relay, electro-hydraulic proportional flow valve etc. The core is 89C2051 MCU. Their mutual relation is shown in Figure 3. If the alcohol content exceeded the standard, the alarm

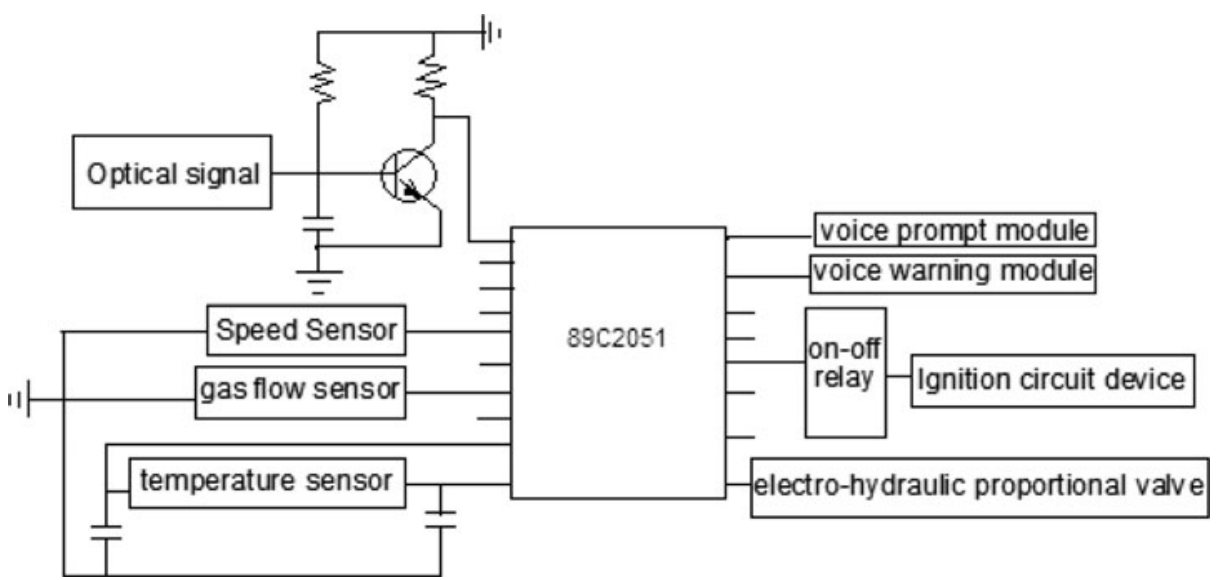

Fig. 3. Drunk driving control circuit system 
module will issue a voice warning and the on-off relay control module will cut off the vehicle's ignition circuit so that the driver can not start the car. If the alcohol content was not exceeded the standard, the voice prompt module will prompt the driver to slow and the electro-hydraulic proportional flow valve will Limit vehicle maximum speed.

\section{Drunk Driving Speed Control System}

The optical signal produced by the alcohol concentration detector is enlarged, then is processed by 89C2051 MCU, is compared with pre-set the alcohol maximum concentration signal. Final the vehicle is controlled accordingly.

If the driver's alcohol concentration measured was higher than the pre-set the alcohol maximum concentration, the 89C2051 MCU will output signal, and supply power to the relay of ignition circuit system. The relay will cut off the ignition system to the engine not be start.

If the driver's alcohol concentration measured was less than pre-set the alcohol maximum concentration and speed measured by speed sensor reached up to the speed needing to control under the alcohol concentration, the 89C2051 MCU will export signal to the drunk driving speed control system so that the vehicle speed can be controlled within the speed that the driver can safely travel under the alcohol concentration.

The drunk driving speed control system mainly consists of accelerator pedal, Spring, hydraulic cylinder, hydraulic control valve, check valve, relief valve, oil tank and some tubing etc. The relationship between the various components is shown in Figure.

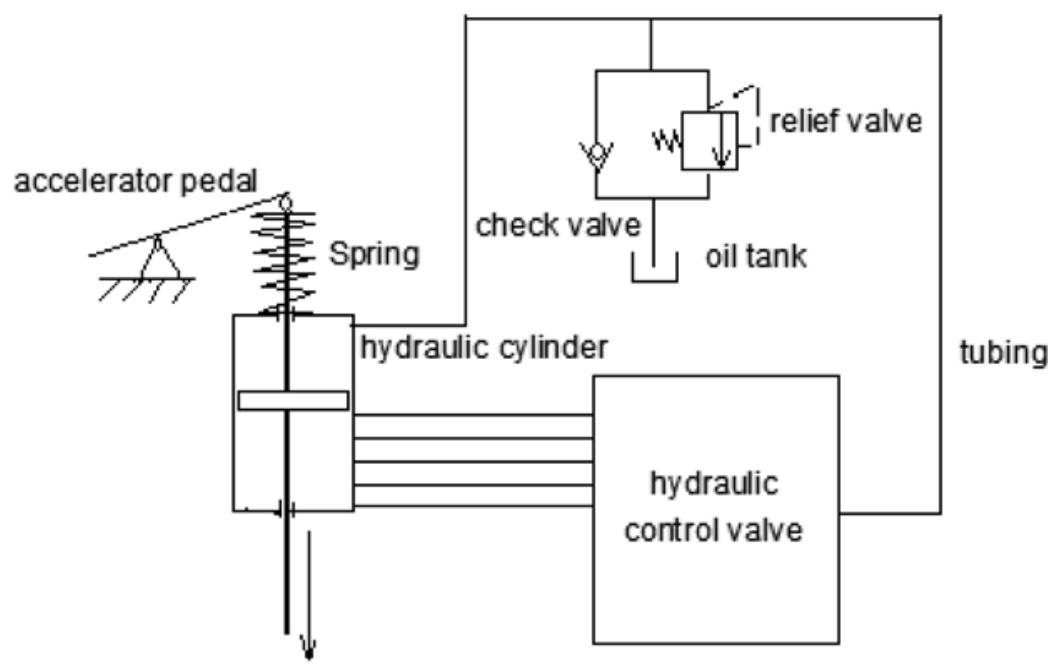

Vehicle throttle control system

Fig. 4. Drunk driving speed control system 
Hydraulic cylinder is a special cylinder. It has some holes in different locations and is connected with the hydraulic control valve by some tubing. Hydraulic control valve is an electro-hydraulic proportional valve. At different currents, the spool of hydraulic control valve can move different distances to seal different the holes. Spring has the role to reset the automobile accelerator pedal. Check valve has a complementary role to oil. Relief valve has a role to set oil pressure.

The control principle of the drunk driving speed control system is that 89C2051 MCU exports corresponding signal to the electro-hydraulic proportional flow valve according to the driver's the alcohol concentration measured, so that its the spool can produce different mobile to seal different holes which connect with hydraulic cylinder, to control the piston of hydraulic cylinder to move different distances, to control the opening size of the vehicle throttle. The size can control vehicle speed.

\section{Summary}

Above the study shows that the online control system to prevent drunk driving can take the initiative to force motor vehicle drivers to test their drinking status, and reasonably control the driver's driving behavior accordingly.

The basic principle is to use the characteristic that $1185 \mathrm{~nm}$ wavelength IR is absorbed by alcohol the most easily to detect the motor vehicle driver's alcohol concentration and to determine the extent of motor vehicle drivers drinking. The alcohol concentration detected is converted into photoelectric signal that is handled by 89C2051 MCU. The signal handled is exported to voice devices to get voice prompts and warning, and exported to the relay of ignition circuit system to cut off the ignition system to control the engine's starting and prevent the driver drunk driving, and exported to electro-hydraulic proportional flow valve to control the maximum speed of motor vehicle according to the driver's alcohol level. The study shows the system can effectively solve the problem to prevent drunk driving and avoid serious traffic accidents due to drunk.

\section{Acknowledgements}

The research was supported by the National Natural Science Foundation of China "The Study of the Principle and tribological design basis of the roll and slide blend bearing in circle pound situation. "(Grant No.51065009).

\section{References}

[1] Feng, J., Zhou, Y., Liu, G., Wu, T.: The optical characteristics of alcohol and alcoholicity detection. Chinese Journal of Spectroscopy Laboratory (March 2006)

[2] Li, Z., Xu, Z., Wang, J.: Active breath alcohol detector. Journal of North China Institute of Aerospace Engineering (October 2009) 